\title{
Criminologie
}

\section{Ils n'ont plus la liberté : réactions à la victimisation et ses conséquences}

\section{Micheline Baril}

Volume 13, numéro 1, 1980

Regards sur la victime

URI : https://id.erudit.org/iderudit/017118ar

DOI : https://doi.org/10.7202/017118ar

Aller au sommaire du numéro

Éditeur(s)

Les Presses de l'Université de Montréal

ISSN

0316-0041 (imprimé)

1492-1367 (numérique)

Découvrir la revue

Citer ce document

Baril, M. (1980). Ils n’ont plus la liberté : réactions à la victimisation et ses conséquences. Criminologie, 13(1), 94-103. https://doi.org/10.7202/017118ar d'utilisation que vous pouvez consulter en ligne.

https://apropos.erudit.org/fr/usagers/politique-dutilisation/ 


\section{ILS N'ONT PLUS LA LIBERTÉ : REACTIONS A LA VICTIMISATION ET SES CONSEQUENCES}

Micheline Baril

Ils n'ont plus la liberté, me disent-ils. L'espace s'est rétréci. Leur vie s'est encombrée d'interdits et de contraintes. Elle est amoindrie. Ce sont la peur et l'angoisse qui les confinent ainsi. Car si la peur est le prix de la liberté, elle en est aussi la limite.

Mais qui sont-ils ? Quarante-deux propriétaires et employés de petits commerces victimes de vols à main armée; également victimes de vols à main armée, deux caissiers de banque et deux particuliers; une jeune femme à qui on a arraché son sac; un témoin de vol qualifié brutalisé durant la perpétration de l'acte ; une victime (secondaire, bien sûr) de meurtre ; une victime d'enlèvement ; deux jeunes femmes violées de façon assez sadique; trois personnes dont le domicile a été cambriolé en leur absence ; une autre qui a surpris son larron la main dans le sac. Leurs témoignages ont été recueillis en entrevues non structurées et enregistrées au magnétophone.

Une première étude a été réalisée durant l'êté 1977 auprès de quarante petits commerçants montréalais ${ }^{2}$. Elle s'inscrivait dans le cadre d'une recherche plus vaste sur les attitudes du public à l'égard de la criminalité. A l'analyse des résultats, l'intérêt a glissé des attitudes à la victimisation elle-même et a donné lieu à une nouvelle analyse qui, à son tour, a orienté une deuxième étude actuellement en cours. Il s'agit, entre autre, de voir si les faits et les émotions observés lors de vols à main armée chez los petits commerçants sont particuliers à ce type d'événements, à ce type de victimes.

On ne trouvera ici ni vision globale de la victimisation ni théorie de la criminalité. L'espace, le temps, et le matériel recueilli à date ne le permettent pas. Ce sujet, je ne l'aborde pas dans son ensemble et on devra voir mes propos comme des hypothèses qui s'appuient sur des expériences vécues. D'ailleurs, je laisserai souvent la parole à mes interlocuteurs.

1. Les petits commerçants victimes de vol à main armée - En quête de justice ", rapport inédit đe recherche effectué par Hélène Grenier et Hélène Manseau sous la direction de Micheline Baril, GRAC, rapport no 5, Université de Montréal. 
Je toucherai quelques conséquences de la victimisation dont on parle peu ou rarement. Si les pertes monétaires et les blessures corporelles subies lors d'événements définis comme criminels attirent de plus en plus l'attention des chercheurs, d'autres suites demeurent passablement méconnues : entre autres, les sentiments de peur, de captivité, d'impuissance. Cette analyse m'amènera à poser quelques questions de base relativement aux définitions de 4 crime », " victime d'acte criminel s, et, implicitement, quant à l'objet de la victimologie et aux fonctions du système pénal ${ }^{2}$.

\section{La peur}

La peur, on la pressent déjà avant l'entrevue. Il n'est pas facile de prendre contact avec les gens qui ont subi un acte criminel grave. Ils ont déménagé, ils ont changé leur emploi, leur numéro de téléphone, ils ont exigé de la police qu'elle ne divulgue leur nom et adresse à quiconque. Lorsqu'on réussit à les rejoindre, bon nombre d'entre eux déclinent l'entrevue, soit qu'ils se méfient d'interviewers considérés comme des agents du système de justice, soit qu'ils craignent que la rencontre ne se solde par une nouvelle victimisation. En entrevue (enregistrée), certaines victimes veillent à ne mentionner aucun nom ou adresse qui ne puisse les identifier avec précision. Ainsi, une dame nous parle de cette ville ou j'habite s, jugeant préférable de taire le nom de cette ville.

Qu'est-ce que la peur? De quoi a-t-on peur? Quand? Pourquoi ? La peur, Maupassant la dit « quelque chose d'effroyable, une sensation atroce, une décomposition de l'âme, un spasme affreux de la pensée et du cœur dont le souvenir seul donne des frissons d'angoisse ${ }^{3} \gg$. Plusieurs personnes ont raconté leur réaction d'épouvante au moment de l'événement et une angoisse qui les tenaille depuis :

a) Sur le coup. - Au moment même de l'agression ou immédiatement après, la plupart des victimes éprouvent de fortes réactions physiologiques et psychologiques qui se manifestent sous des formes variées : paralysie, palpitations, hystérie, tremblements. Là où il y a armes, cagoules, brutalités ou menaces verbales, de telles

2. Micheline Baril. New Directions in Victim Research : Victims' Perceptions of Crime and the Criminal Justice System, 1978, Unpublished M. Phil. thesis, Cambridge, University of Cambridge.

Ed. 1908.

3. Guy de Maupassant, a Contes de la Bécasse ?, CEuvres complètes, 
réactions ne surprennent guère. Parfois, on n'a pas conscience de l'objet de sa peur, on est tout simplement possédé par elle. Parfois, la crainte est focalisée sur des points précis : la douleur physique et la mort.

J'étais sûr qu'il allait me tirer.

J'essayais de pas crier parce que je savais qu'il allait me tuer. Je me concentrais là-dessus : crie pas, panique pas, ça va venir à finir. Me tuer vite, ça ne me dérangeait plus beaucoup mais j'avais peur qu'il se mette encore à me battre.

Dans trois cas de cambriolage, il n'y a eu aucun contact entre voleur et volé. Pourtant, la peur ressentie en constatant l'intrusion a été semblable, moins intense assurément, à celle vécue par les personnes physiquement confrontées à leurs agresseurs.

La réaction que j'ai eue, ça a été de sortir de la maison, pas rester dans la maison. Je voulais qu'il y ait une tournée de la maison qui soit faite avant. Je suis allée chez les voisins, j'ai sonné à la porte, je suis arrivée là toute tremblante. On n'imagine pas avant que ça arrive qu'on puisse trembler pour une chose comme ça, parce qu'en fait, moi, j'ai vu personne.

$\mathrm{Au}$ fond, ce qui effraie autant, sinon davantage que le danger réel, c'est la surprise et l'incertitude, c'est l'absence momentanée de contrôle sur une situation vitale.

b) Après coup. - Avec le temps, on voit cette peur se muer en angoisse, c'est-à-dire un sentiment d'insécurité, une appréhension du futur, l'impression d'un danger dont on ne peut se prévenir parce qu'on ne sait pas où, quand et comment il va frapper.

"S'ils reviennent », "si ça se produit encore », "la prochaine fois 》, répètent nos interlocuteurs. Et avec raison puisque la plupart d'entre eux ont été victimisés plus d'une fois. Les quarante petits commerçants interviewés lors de la première étude avaient subi, au total, environ 250 vols à main armée, soit plus de cinq par individu, en moyenne. Une caissière de banque a fait l'expérience de cinq vols à main armée en trois ans. Les banques et les petits commerces sont, naturellement, des cibles de choix. Toutefois, ce même phénomène de répétition a été observé dans le cambriolage de résidences. Une dame l'explique ainsi :

Mon mari m'a dit : « Ils vont peut-être revenir mais qu'est-ce que tu veux qu'on y fasse ? Quand on y va une fois quel- 
que part, on y retoume. Je trouve ça bien logique aussi. Peut-être quand on sait comment c'est dans une maison, on sait ce qu'on a vu, quoique, comme je disais, y a pas de choses plus précieuses ici qu'ailleurs, je vois absolument pas ce qui est plus intéressant ici, mais enfin, on sait qu'il y a telle ou telle chose. Puis, ça a marché une fois.

Ce n'est pas seulement la répétition de l'agression subie qu'on redoute mais toute forme d'agression. Par exemple, une personne dont le domicile a été cambriolé, cesse de sortir seule le soir. Plusieurs interlocuteurs ont aussi exprimé des réticences à identifier leurs assaillants ou à témoigner devant le tribunal, de crainte de faire l'objet de représailles.

c) Une autre fois. - Avec le temps, on s'habitue? Plusieurs personnes ont mentionné que leurs réactions avaient été moins intenses, la deuxième ou la troisième fois. Ou bien, on se résigne. Ça fait partie du métier, c'est la vie, on n'y peut rien. Ou bien, on s'administre une thérapie de cuirassement. Ou encore, on décide de lutter activement contre la situation, en s'armant, par exemple. Il ne faut pas oublier toutefois que les résistants sont sur-représentés dans nos échantillons qui sont composés, en presque totalité, de personnes qui n'ont pas encore quitté l'emploi ou la demeure où l'événement a eu lieu; la fuite est, en effet, une autre forme de solution. De plus, il semble que la situation soit différente chez le personnel des banques où les chocs nerveux et les crises d'hystérie seraient le fait des personnes qui ont vécu plusieurs expériences de hold-up.

d) Il ne faut rien exagérer. - Chaque personne a sa façon bien à elle de vivre une expérience traumatisante. Certains sont terrorisés par des événements plutôt bénins alors que d'autres conservent leur sang-froid devant de grands périls. Les uns réagissent violemment sous l'effet du choc mais récupèrent rapidement par la suite; les autres ne s'effondrent qu'une fois le danger disparu. Mais force nous est ici de simplifier la description.

\section{Une liberté entravée}

Face à une arme, ligoté ou enfermé dans un frigo, on n'éprouve sans doute pas une grande liberté de mouvement. Mais ce que je veux rapporter maintenant, ce sont ces contraintes de l'après que nous ont décrites nos interlocuteurs. Elles découlent de l'angoisse et des précautions adoptées pour prévenir une nouvelle victimisation. 
Ca a créé chez moi beaucoup de révolte parce que je me suis dit je ne peux plus vivre comme auparavant. Ça m'enlève beaucoup de liberté. Je ne suis jamais sortie seule le soir depuis, je ne pourrai plus prendre des cours du soir. Il me semble qu'avant, j'avais une vie plus..., j'étais plus libre de faire ce que je voulais.

Chaque fois que j'entre, $j$ 'entre dans la maison très lentement. J'écoute avant parce que je mets le son chaque fois que je pars. En entrant, j'écoute. Si le son est là, c'est assez normal. $\mathrm{Y}$ a pas une fois que j'entre dans la maison de façon libre.

Une personne ne se déplace plus seule, une autre porte une arme. Telle autre n'ouvre plus sa porte à moins qu'un visiteur ne se soit annoncé. Quelqu'un a déménagé. Une jeune fille ne peut plus vivre seule. Un caissier a laissé son emploi. Une victime indirecte n'ose plus aller faire ses transactions bancaires ellemême. Tous ont eu recours à de quelconques dispositifs de sécurité : verrous, serrures spéciales, clochettes, systèmes d'alarme, etc. Les précautions sont nombreuses et variées. Outre leur coût, les restrictions de mouvements qu'elles impliquent, les crispations qu'elles suscitent et expriment, elles représentent une perte de temps que nos interlocuteurs estiment considérable.

L'argent, c'est rien. J'avais à peu près $\$ 5.00$. Et même si j'en avais eu plus. Ce qui est le pire, sur le coup, c'est se faire attaquer et ensuite, c'est la perte de temps. J'ai eu des emmerdements pendant deux mois après. Pour commencer, j'avais pas mes clés pour rentrer à la maison. On est comme paralysé, handicapé je devrais dire, sans permis de conduire, sans carte d'identité, sans rien, sans compter qu'on se demande ce qu'il va faire avec ça, lui. J'ai perdu beaucoup de temps.

On préférerait utiliser notre temps à d'autre chose que d'essayer de protéger la maison, franchement. Je n'ai plus la liberté, pas de mon temps, même pas de ma maison, c'est ça qui me révolte. Mais on se dit comment ça se fait que nous, on veut pas déranger personne puis on fait notre vie comme ça, dans notre maison, puis tout à coup, des gens décident de venir nous déranger, à ce point-là, puis venir s'immiscer dans toutes nos affaires personnelles, d'aller fouiller dans tous nos tiroirs, puis de tout sortir, de tout mettre en désordre. C'est peut-être très bénin ça, mais je ne m'étais jamais représenté ça, mais tout à coup, on entre dans notre maison et c'est comme si un coup de vent avait passé.

Ces citations se passent de commentaires. Il serait bon toutefois de souligner que le thème de l'intrusion apparaît dans 
presque toutes les entrevues, quel que soit le type d'événement subi. Ce dernier semble vécu surtout comme une violation de soi ou de son territoire.

\section{L'impuissance}

Déjà dans l'étude auprès des petits commerçants, la constatation qui s'imposait avec le plus de force à l'analyse des résultats, était l'impuissance vécue face au vol à main armée, à l'éventualité d'une nouvelle victimisation et aux suites de tels incidents. Loin de dissiper cette impression, l'étude en cours actuellement la renforce.

a) Face à l'événement. - C'est dans les cas de viol étudiés que l'impuissance ressort avec le plus d'acuité. Une jeune femme, nommons-la Louise, a été assaillie chez elle alors qu'elle venait de se mettre au lit, par un individu qui s'était embusqué dans quelque coin de la maison. Profitant de la surprise, de l'obscurité, il l'a d'abord blessée gravement et menacée de mort avant de la violer et sodomiser durant environ deux heures. Plus morte que vive, Louise n'avait alors conscience que de la douleur, la peur, la nécessité de se laisser faire pour survivre peut-être. Plus tard, durant sa convalescence, l'iđée qui la hantait, c'était - j'emploie sa propre expression - sa lâcheté. Elle n'avait pas défendu son intégrité. Au moment de l'acte, elle n'avait pensé qu'à survivre, me dit-elle, mais plus tard elle se demanda si c'était bien la peine de survivre au prix de la perte de soi. Elle se décrivait ainsi : «Je suis comme infirme. » Louise me rappelle les propos d'un journaliste anglais qui, à sa troisième victimisation en huit ans, avait riposté physiquement sur la personne de son agresseur : "Même si, le lendemain matin, j'étais incapable d'ouvrir mon œil, je reposais au lit avec un sentiment d'exaltation. J'avais défendu mon intégrité ${ }^{4}$.

Fairlie dit plus loin, se référant à la première attaque, un cambriolage cette fois : «J'avais moins l'impression d'avoir été volé que violé ${ }^{5}$.

4. Henry Fairlie : * The exhilaration of violence ", Montreal Star, 6 mai 1978 : A Although I could not open my eye the next morning, I lay in bed with a feeling of exhilaration. I had defended my integrity. $\rightarrow$ raped.

5. Idem : .... I felt less that I had been robbed that I had been 
Nous avions déjà observé, chez les petits commerçants, cette fierté d'avoir résisté ou déjoué l'adversaire un tant soit peu. Par contre, chez les hommes en particulier, la non-résistance tendait à s'accompagner d'un sentiment de dévalorisation personnelle.

Le sentiment d'impuissance s'avère beaucoup plus dévastateur, dans certains cas, que les conséquences visibles de l'événement. C'est une diminution de l'estime de soi, une humiliation, un vague remords, l'impression d'avoir été utilisé, le sentiment de n'être plus entièrement soi-même.

b) Face au futur. - Il existe déjà une littérature assez abondante sur l'agresseur malgré lui ou la victime provocatrice. La * victime négligente » est aussi un concept assez familier. Mes échantillons, non représentatifs, ne renferment aucun cas auquel on puisse apposer ces étiquettes. Non pas que je dénie l'existence de telles situations. Elles existent bel et bien. A ce stade-ci, il m'a semblé préférable de ne considérer que les situations où il n'y avait eu aucune incitation ou provocation visible de la part de la victime.

Nous l'avons vu, les victimes craignent une nouvelle agression et avec raison. Peuvent-elles se protéger? Sont-elles prédisposées par quelque caractéristique à vivre de tels drames? A cette dernière question, on peut répondre affirmativement pour la plupart des cas. Leur mode de vie ou leur type d'occupation les prédispose. Pour elles, la seule mesure de protection efficace est le changement de résidence, de routine ou d'emploi. Une caissière de banque, par exemple, ou le propriétaire d'un petit commerce de quartier, doivent considérer l'agression violente comme un des risques du métier et aucune mesure de protection ne diminuera sensiblement ce risque.

Mais je crois aussi, qu'une première victimisation prédispose à d'autres agressions du même genre. J'ai cité plus haut cette dame qui exprimait une telle opinion : les voleurs connaissent maintenant la disposition des lieux, les habitudes et les réactions des gens, les gains probables; l'opération s'est déroulée sans anicroches une première fois. Il faut bien voir que l'agresseur est aussi effrayé de l'inconnu que sa victime. C'est d'ailleurs l'opinion de policiers que j'ai consultés à ce sujet. Selon eux, les criminels tendent à reproduire un modus operandi qui s'est déjà avéré efficace. Cette répétition pourrait fort bien s'étendre au choix des victimes. Un petit commerçant qui a reconnu ses agresseurs non 
masqués s'était d'ailleurs exclamé : «Pas encore vous autres? \$ Mais oui, a-t-on répliqué, la dernière fois, ça a été payant. Un autre propriétaire de petit commerce estimait que la seule façon de faire cesser les attaques est de résister ou de contre-attaquer. * Autrement, ça se sait, un tel à telle place, c'est un bon spot. Le gars se laisse faire.

Si mon hypothèse se vérifiait, la seule solution efficace au problème des victimisations multiples serait, encore une fois, la fuite. A moins qu'on ne considère la violence comme solution efficace...

c) Face aux suites, - Le plus souvent, l'événement déborde le cadre d'une brève confrontation entre deux protagonistes. D'autres agences entrent sur scène dès que le rideau tombe sur le drame initial : hôpital, compagnie d'assurances, employeurs, presse, police, tribunaux.

L'hôpital va signaler le cas à la police, si ce n'est déjà fait, l'assurance exige un rapport de la police. Dans le cas d'une banque, l'employeur a sa propre police qui agit, dans une certaine mesure, comme intermédiaire entre le caissier attaqué et la police officielle et émet ses propres directives. S'il s'agit d'une affaire sanglante ou autrement spectaculaire, la presse, branchée à l'écoute de la radio de police, traquera la pauvre victime jusque dans son repaire, pour un mot, une photo.

Dès que l'une ou l'autre de ces agences intervient, la victime se voit dépouillée de ce qui était jusque-là sa tragédie personnelle. Désormais, l'affaire est publique. Selon nos interlocuteurs, le sentiment d'impuissance qu'ils ont vécu au moment de l'événement s'intensifie lors de l'intervention. Non seulement ils n'ont pas reçu l'aide qu'ils avaient espérée mais ils se sont senti utilisés comme objets vers des fins qui n'étaient pas les leurs.

Fait que là, si je regarde ça, grosso modo, toutes les interactions qu'il peut y avoir dans une histoire comme ça, d'abord les agresseurs, je les ai jamais vus. Les gens qui travaillent à la banque, la police puis les journalistes, je me suis fait une opinion à ce moment-là. Les journalistes, je les aime pas, face à l'expérience que j'ai eue. Je les avais déjà aimés mais là, je les ai très loin. Les policiers, je trouve que, bon, ils font leur job, ils font leur boulot. Puis, je trouve que l'administration, les gros boss de la banque, face à une situation comme ça, ils réagissent en fonction de signes de 
piastres. [...] Je me suis rendu compte qu'à ce moment-là, j'étais un peu comme un outil involontaire pour des gens qui menaient une certaine enquête.

Ça m'a révolté. Ce que j'ai trouvé de pire dans tout ça, c'est de servir de cobaye. Ça me révolte encore. Je me suis senti manipulé par les événements puis par les gens.

L'expérience dont il est question est un enlèvement avec tentative d'extorsion.

Près de la moitié des victimes interviewées ont eu affaire au système de justice criminelle au sujet de l'une ou l'autre des expériences qu'elles avaient vécues. Précisons que l'intervention première de la police n'est pas considérée par nos interlocuteurs comme un contact avec le système mais plutôt comme un secours d'urgence, d'ailleurs bien coté. L'enquête subséquente, la poursuite, le procès, la condamnation, s'ils ont lieu, appartiennent au système. Et ce système est jugé de façon très négative. Au mieux, c'est une comédie; au pire, c'est une sombre conspiration des puissants contre les faibles. Et nos victimes y ont fait l'amer constat de leur impuissance. Elles sont souvent forcées de faire rapport à la police ; les compagnies d'assurance l'exigent.

Une fois que le «crime» est connu de la police, la victime n'a plus aucun contrôle sur la suite. Elle est supposée avoir porté plainte, qu'elle en ait été consciente ou non et elle ne peut retirer cette plainte. Devant le tribunal, elle devient un témoin qu'on interrogera si son témoignage peut servir les fins de la poursuite ou de la défense. Elle est rarement informée du verdict et de la sentence.

Tous nos interlocuteurs ont vécu ce contact avec le système judiciaire comme une perte de temps et un événement qui se déroulait à l'extérieur d'eux mais dont ils étaient le prétexte :

Ils s'imaginent peut-être qu'on est des fous, qu'on se rend pas compte que c'est une comédie. Ça crève les yeux qu'ils se sont arrangés d'avance. Ils s'entendent, le juge, les avocats, avant d'entrer. Alors, moi, je vois vraiment pas pourquoi ils nous font poirotter comme ça, on serait pas là puis ça ne changerait rien.

Si cette personne s'est sentie inutile, d'autres ont eu l'impression d'aider des agents de la justice à faire leur boulot et ont déploré le fait que ces agents ne leur aient pas témoigné une certaine considération en retour de leur assistance. 
Le discours des personnes interviewées soulève de nombreuses interrogations quant aux fonctions du système de justice criminelle et quant aux implications de la définition de certaines conduites comme criminelles. Telles que vécues par les individus, ces conduites seraient des conflits d'intérêts, de besoins humains et les parties lésées s'attendent à ce que le système de justice joue un rôle d'arbitre, ce en quoi elles sont déçues.

Tout compte fait, le terme « victime d'acte criminel \& m'apparaît une fiction. L'événement lui-même provoque des réactions et des conséquences, pénibles certes, mais somme toute très semblables à celles qui sont déclenchées par nombre de situations traumatisantes, violentes, quoique non définies comme criminelles. Les victimes, d'ailleurs, ne qualifient jamais les evénements vécus de crimes, ni leurs agresseurs de criminels. Ce qui pourrait faire la spécificité d'un acte criminel, tel que vécu par la partie passive, c'est l'intervention du système judiciaire. Or, dès que l'affaire est portée à la connaissance du système, elle échappe à la victime qui dès lors est définie comme plaignant puis, comme témoin. L'affaire est maintenant criminelle, soit, mais la victime n'est plus « victime », c'est l'État (ou la société) qui est victime. 